\title{
Testing of submicrometer fluorescence microprobe based on Bragg-Fresnel crystal optics at the ESRF
}

Cite as: Review of Scientific Instruments 66, 1461 (1995); https://doi.org/10.1063/1.1145941

Published Online: 04 June 1998

A. Snigirev, I. Snigireva, P. Engström, S. Lequien, A. Suvorov, Ya. Hartman, P. Chevallier, M. Idir, F. Legrand, G. Soullie, and S. Engrand

\section{ARTICLES YOU MAY BE INTERESTED IN}

Hard $x$-ray phase zone plate fabricated by lithographic techniques

Applied Physics Letters 61, 1877 (1992); https://doi.org/10.1063/1.108400

Hard x-ray microprobe with total-reflection mirrors

Review of Scientific Instruments 63, 578 (1992); https://doi.org/10.1063/1.1142710

The recent development of Bragg-Fresnel crystal optics. Experiments and applications at the ESRF (invited)

Review of Scientific Instruments 66, 2053 (1995); https://doi.org/10.1063/1.1145726

\section{AlP Author Services}
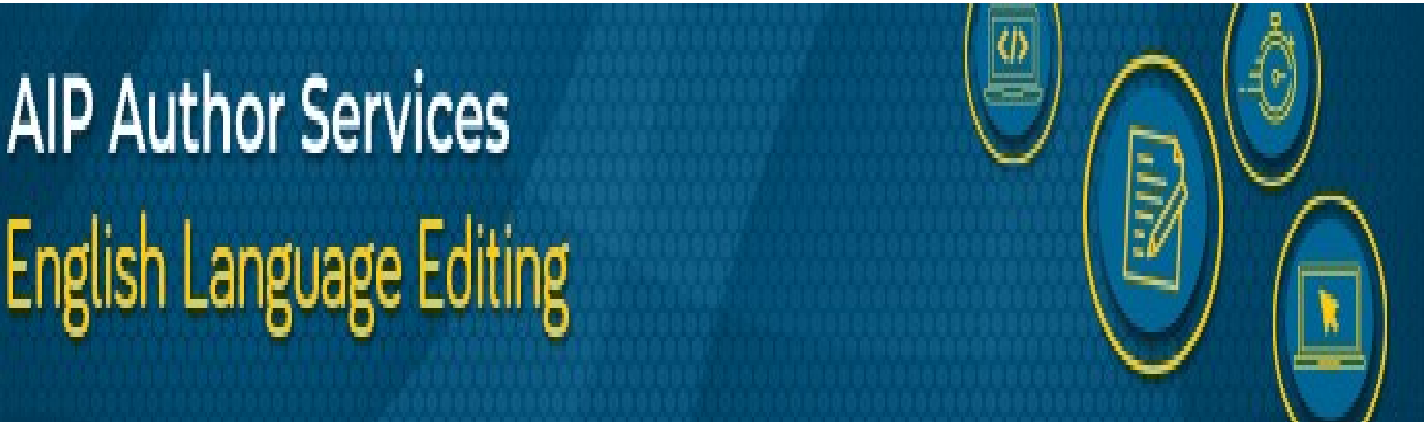


\title{
Testing of submicrometer fluorescence microprobe based on Bragg-Fresnel crystal optics at the ESRF
}

\author{
A. Snigirev, I. Snigireva, P. Engström, and S. Lequien \\ ESRF, B.P. 220, F-38043 Grenoble, France
}

\begin{abstract}
A. Suvorov and Ya. Hartman
Institute for Microelectronics Technology, Russian Academy of Sciences, 142342 Chernogolovka, Moscow Region, Russia
\end{abstract}

P. Chevallier, M. Idir, and F. Legrand

LURE, Universite Paris-Sud, Bat. 209D, F-91405 Orsay, France

G. Soullie

Laboratoire de Chemie-Physique, Universite Pierre et Marie Curie, F-75005 Paris, France

\section{S. Engrand \\ CSNSM, Universite Paris-Sud, F-91405 Orsay, France}

(Presented on 18 July 1994)

\begin{abstract}
An x-ray fluorescence microprobe with circular Bragg-Fresnel lens was tested at the European Synchrotron Radiation Facility Microfocus beamline. A focal spot of $0.7 \mu \mathrm{m}$ was observed using the knife-edge technique at the energy $7.6 \mathrm{keV}$ (wavelength $1.6 \AA$ ). The intensity in the focal spot was measured to $10^{8} \mathrm{ph} / \mathrm{s}$ in energy bandwidth of $10^{-5}$. The fluorescence microprobe was applied for mapping $100 \mu \mathrm{m}$ size micrometeorite at $13.5 \mathrm{keV}$. (C) 1995 American Institute of Physics.
\end{abstract}

\section{INTRODUCTION}

Recent experiments of $\mathrm{x}$-ray focusing by single crystal Bragg-Fresnel lenses $(\mathrm{BFL})^{1-5}$ have shown the real perspective of developing microprobe techniques on the basis of Bragg-Fresnel optics for microdiffraction ${ }^{4}$ and microfluorescence. ${ }^{5}$ The high brilliance and the coherence of an $\mathrm{x}$-ray beams provided by a third generation of synchrotron radiation sources like ESRF make it possible to reach a submicrometer resolution: $10^{8} \mathrm{ph} / \mathrm{s}$ was measured in $0.8 \times 40$ $\mu \mathrm{m}^{2}$ focal line with linear BFL. ${ }^{5}$ Linear microprobe has a number of attractive features including energy tunability in the range from 2 to $60 \mathrm{keV}^{6}$ Nevertheless, a number of diagnostic problems requires a $2 \mathrm{D}$ collimation of the $\mathrm{x}$-ray beam. This can be achieved by applying two linear BFLs in cross setup ${ }^{1,2}$ or by using circular BFL in backscattering geometry. ${ }^{3,4}$ In this paper a 2D microprobe based on circular BFL was tested in order to examine the resolution and to study applicability for microtluorescence..Compared with the cross geometry of two linear BFLs, a circular BFL has an evident advantage of using simpler mechanics for BFL stage, since a single optical element (circular BFL) do not need any accurate angular positioning, which is the case for the cross geometry setup. Due to focusing in two directions one can expect better signal to noise ratio for the circular BFL compared to the linear one.

\section{EXPERIMENT AND RESULTS}

The performance of the fluorescence microprobe base on Bragg-Fresnel lens was tested at the ESRF Microfocus beamline (Fig. 1). A low- $\beta$ undulator with the source size $130 \times 90 \mu \mathrm{m}^{2}$ was located at a distance of $35 \mathrm{~m}$ from the BFL. The 7.6 and $13.5 \mathrm{keV}$ monochromatic beam was selected by an $\mathrm{N}_{2}$-cooled channel-cut $\mathrm{Si}$ monochromator and was focused on a sample by a circular Ge BFL using 444 and 777 reflections in nearly backscattering geometry.

A circular phase BFL was fabricated on a Ge-111 substrate. The basic geometrical parameters of the BFL are: an outermost zone width of $0.15 \mu \mathrm{m}$, the height of the structure of $2 \mu \mathrm{m}$, and an aperture of $100 \mu \mathrm{m}$ for $7.6 \mathrm{keV}$ and $150 \mu \mathrm{m}$ for $13.5 \mathrm{keV}$ (Fig. 2). The BFL was mounted on an $X Y Z$ translation stage with a precise goniometer module, with horizontal axis of rotation, to allow an adjustment of the Bragg angle. A $70 \mu \mathrm{m}$ pinhole was placed downstream of the lens just before the specimen to avoid the background radiation from the flat area of the Ge substrate.

A small $\mathrm{He} / \mathrm{Ne}$ (red) laser was adjusted to simulate the
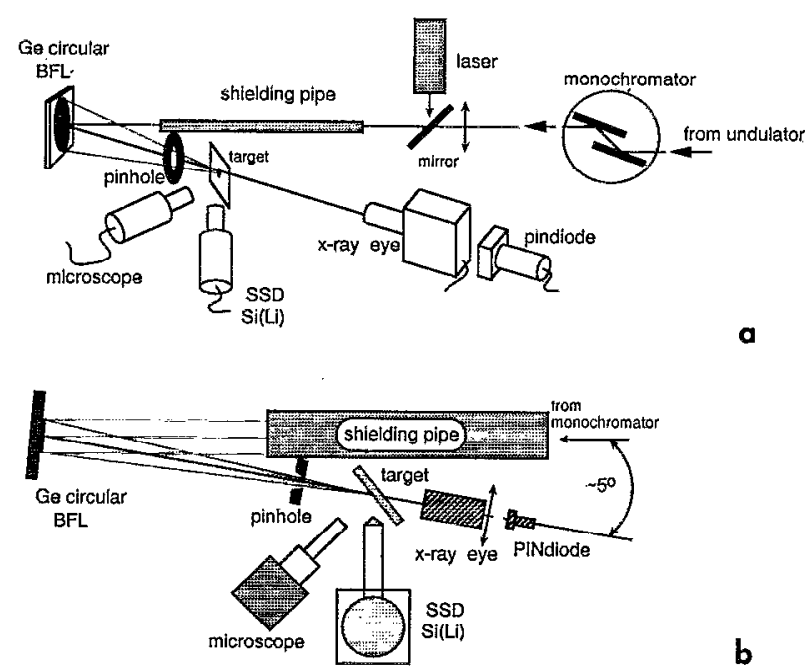

b

FIG. 1. Schernatic display of the experimental setup. Focal distance, $F=10$ $\mathrm{cm}$ for energy, $E=7.6 \mathrm{keV}(\lambda=1.6 \AA)$ (a) and top view (b). 


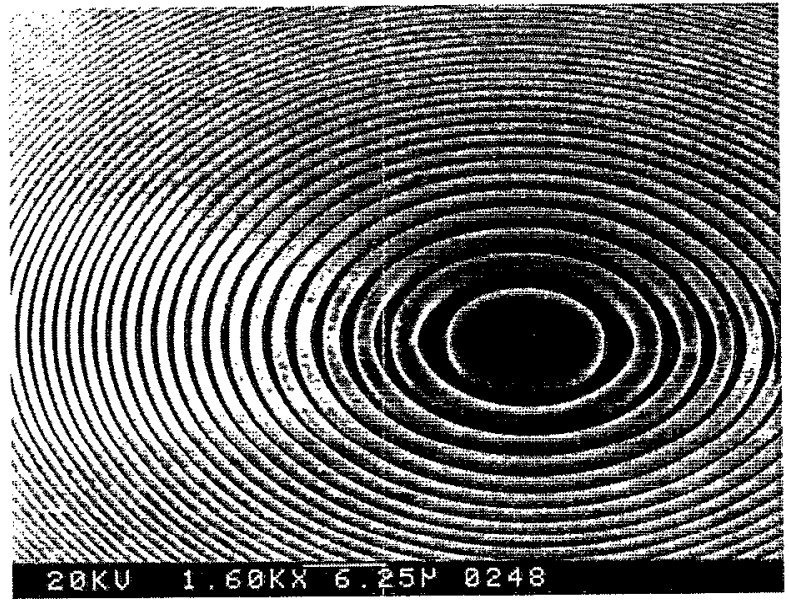

FIG. 2. SEM photograph of the circular BFL.

direction of the $x$-rays beam to perform an easy preliminary alignment of the lens. A " $x$-ray eye camera," Si PIN photodiode and Kodak high-resolution films were used for final adjustment of the setup. In Fig. 3 one can see the $x$-ray image of the focused beam taken with a high-resolution Kodak film placed a few millimeters behind the sample position (focal plane). The central high intensity focal spot is surrounded by the geometric shadow of the BFL aperture region, where the intensity is reduced substantially because only a small fraction of the incident beam is delivered to the zero diffraction order for the phase BFL. The intensity beyond the BFL shadow is due to reflection by the flat $\mathrm{Ge}$ crystal outside of the BFL area.

The sample was mounted on a three-axes $X Y Z$ remote controlled sample stage built from 3 Microcontrole translators with a $0.1 \mu \mathrm{m}$ steps length, located on the focal distance of $10 \mathrm{~cm}$ from the BFL. A Caleo video color optical microscope (magnification 700) was used to locate the sample in

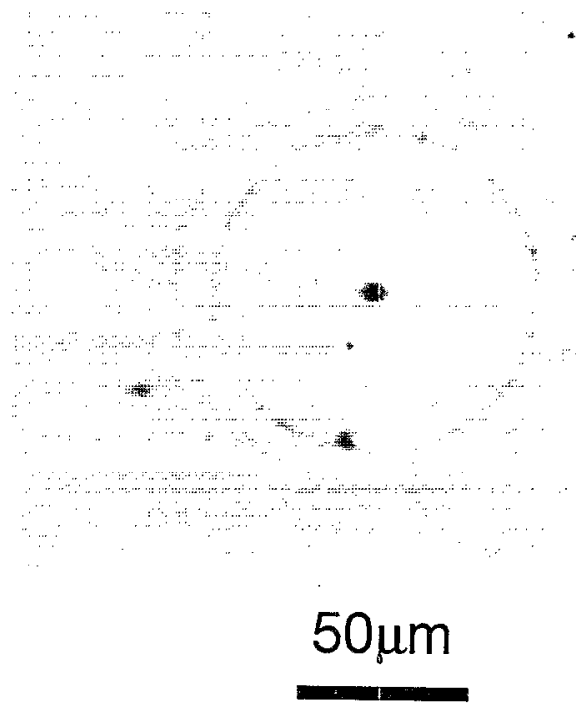

FIG. 3. X-ray image of the focused beam.

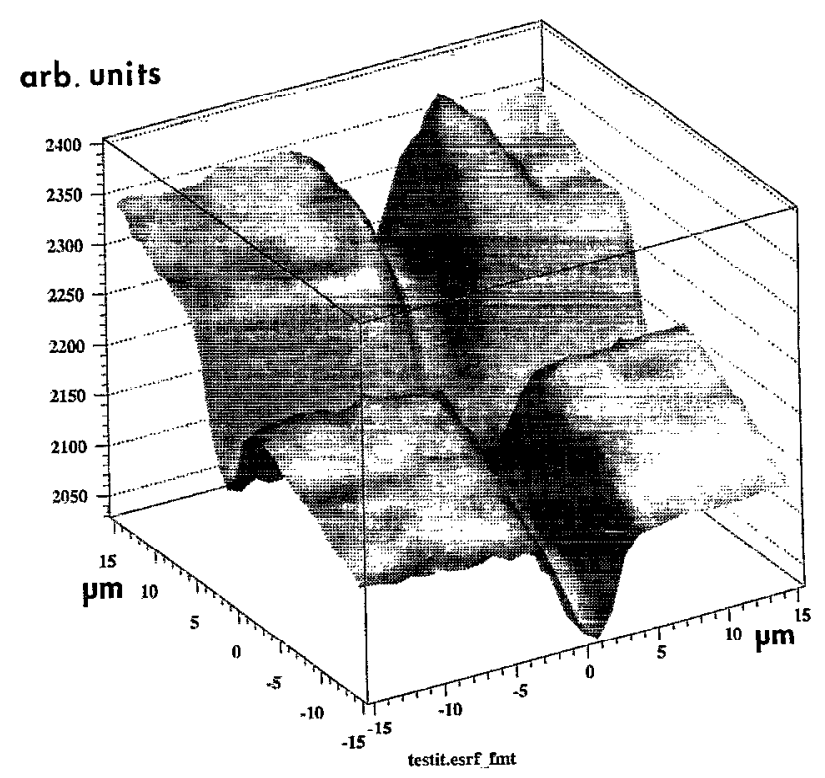

FIG. 4. The distribution of the transmission intensity mapped by scanning the $4 \mu \mathrm{m} \mathrm{W}$-wire cross through the $\mathrm{x}$-ray $(7.6 \mathrm{kcV})$ beam.

the beam and to identify the region of interest on the sample. Adjustment of the sample to the $\mathrm{x}$-ray focal spot was done as follows. First, the W-wire $4 \mu \mathrm{m}$ cross placed instead of the sample, was adjusted to the focused $x$-ray beam, using the drastic decreasing in the intensity of the $x$-ray beam measured by PIN photodiode. A $4 \mu \mathrm{m}$ W-wire cross was scanned through the beam, the transmission intensity profile is presented in the Fig. 4. Second, the hairs cross in the microscope are set to the $\mathrm{W}$-wire cross. Finally, the sample $(\mathrm{Cr}$ mask) replaces the $\mathrm{W}$-wire cross and the analysis point on it is set to the hairs cross. The analysis point can be set to the $\mathrm{x}$-ray focal spot within a few microns.

A Cr mask MRS-2 (magnification reference standard) from Geller Microanalytical Laboratory with a chromium layer of $80 \mathrm{~nm}$ thickness was used as a test object in order to measure the resolution and the sensitivity of the microprobe.

The fluorescence signal was recorded with a Princeton Gamma Teek $\mathrm{Si}(\mathrm{Li})$ detector of $13 \mathrm{~mm}^{2}$ area and $148 \mathrm{eV}$ energy resolution for $5.9 \mathrm{keV}$ photons. A collimator was set in the axis of the detector to ensure that only those photons arising from the sample can enter its active area. To subpress further background due to scattering the detector was mounted in the plane of the synchrotron ring and at $90^{\circ}$ to the incident beam. Due to the high horizontal polarization of the synchrotron light these geometrical conditions reduce the Rayleigh and the Compton scattering from the sample to a minimum. Lead shielding was also used around the detector to decrease the background due to scattering of the incoming beam through its air path to the sample.

Pulses from the detector were processed through a Tennelec TC 144 amplifier operating in the triangular shaping mode. The pile up rejection output was sent to a pulse high analyzer that is a Nucleus PCA card installed in an IBM PC-AT3 computer.

In order to synchronize and to control the sample move- 


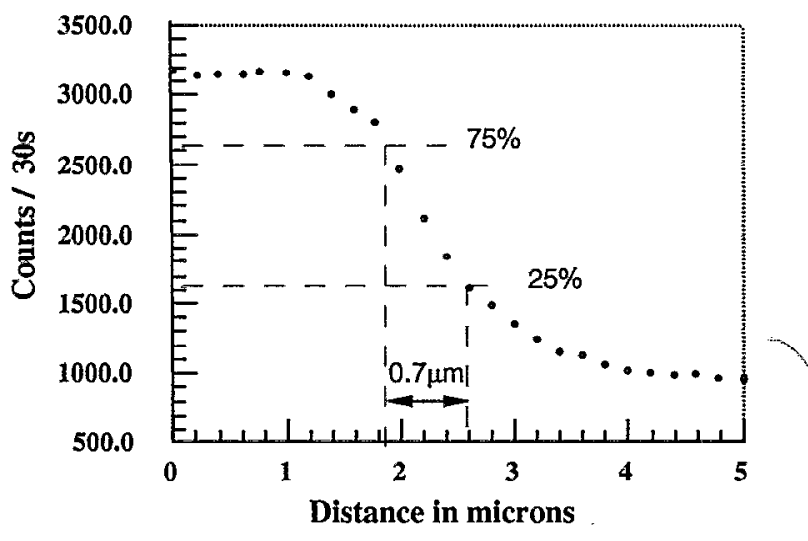

FIG. 5. Experimental variation of $\mathrm{Cr} K_{\alpha}$ fluorescence signal upon scanning a Cr-strip pattern.

ments and data collection a PC-based software package called PILOT, developed by LURE, was uscd. To reduce the amount of data from a scan the software saves the regions of interest (ROI) for each point, instead of saving the full spectrum, for up to 10 ROIs. This also reduces the time of data treatment in sacrifice of the precision in peak analysis, e.g., tails of neighboring large peaks can introduce systematic errors in small peak areas.

Figure 5 shows the sum of a scan across one of the $\mathrm{Cr}$ strips. The step width was about $0.1 \mu \mathrm{m}$. The counting time per point was $30 \mathrm{~s}$. The background of about $70 \mathrm{c} / \mathrm{s}$ is due to secondary generated fluorescence of neighboring $\mathrm{Cr}$ strips. The $25 \%$ and $75 \%$ markers indicate the effective $\mathrm{x}$-ray imaging probe dimension. The knife-edge scan data shows a focal spot of $0.7 \mu \mathrm{m}$. The intensity in the focal spot was measured to $10^{8} \mathrm{ph} / \mathrm{s}$.

The developed submicrometer fluorescence microprobe was applied for elemental analysis of small (10 to $40 \mu \mathrm{m})$ inclusions in micrometeorites collected in Antarctica, by melting and filtering large amounts of the blue ice. These micrometeorites are mainly composed of desequilibrated assemblages of pyroxen and olivin phases (iron and magnesium silicates). In about $60 \%$ of these specimens a new phase mainly composed of carbon, oxygen, phosphor, and sulfur has been discovered. Due to its major elemental composi-

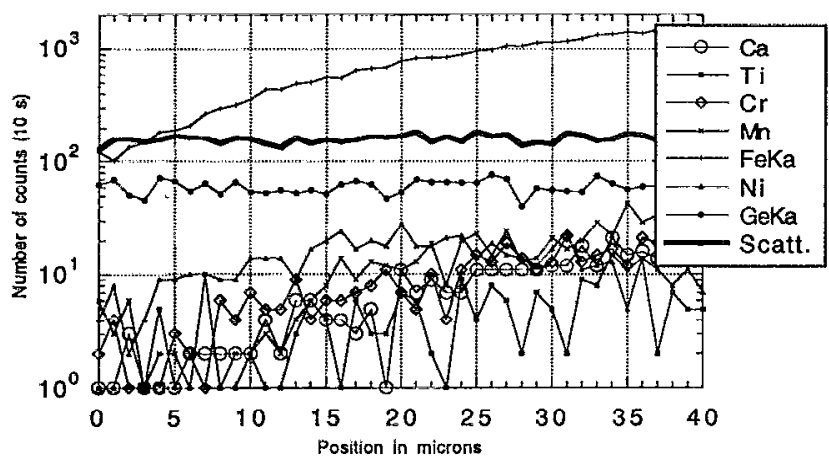

FIG. 6. Experimental fluorescence spectrum of COPS inclusion vs the position on the sample. tion, this new phase has been called COPS. The formation of this phase is not yet understood and trace element analysis could help to determine the origin of its material.

One of the meteorite was inbedded in epoxy and polished until a COPS inclusion appeared in view. The micrometeorite was studied at $13.5 \mathrm{keV}$ using a submicrometer sized beam from a BFL.

A line scan through the COPS inclusion was performed with $1 \mu \mathrm{m}$ steps. Each spectrum was collected for $10 \mathrm{~s}$ and ROI corresponding to $\mathrm{Ca}, \mathrm{Ti}, \mathrm{Cr}, \mathrm{Fe}, \mathrm{Ni}, \mathrm{Ge}$, and scattered peak $(13.5 \mathrm{keV})$ were recorded. Results are shown in Fig. 6 where the count rate in each ROI is plotted versus the position on the sample. As can be seen in Fig. $6 \mathrm{Fe}$ has an increase in concentration going from the edge to the other edge, whereas the other elements are constant.

The data shown in Fig. 6 are significant for the various elements, because the background due to scattering was very low in this energy region. Further if some of the data where due to the low energy tail of, e.g., the strong Fe peak this contribution should follow the same variation as the iron peak which is not the case.

\section{CONCLUSION}

Two-dimcnsional sub- $\mu \mathrm{m}$ fluorescence microprobe has been demonstrated for the first time for the combination of a Bragg-Fresnel lens and a high brilliance undulator at a third generation SR source. The fluorescence knife-edge measurement shows with high accuracy the spatial resolution of the circular Bragg-Fresnel lens that is about $0.7 \mu \mathrm{m}$. A flux of $10^{8}$ photons per $s$ in a focal spot was achieved in very narrow bandpath $10^{-5}$. In the case when a resolution of one or a few microns is sufficient and a higher flux is needed the elliptical multilayer BFL can be applied. ${ }^{7}$

\section{ACKNOWLEDGMENTS}

We gratefully acknowledge P. Dhez and A. Freund for the help and advice; C. Riekel for providing beam-timc at the Microfocus beamline; M. Kocsis for technical support. The authors would like to thank V. Yunkin, C. David, B. Kaulich for Ge-BFL fabrication.

${ }^{1}$ U. Bonse, C. Riekel, and A. A. Snigirev, Rev. Sci. Instrum. 63, 622 (1992).

${ }^{2}$ V. V. Aristov, Yu. A. Basov, Ya. M. Hartman, A. A. Snigirev, and C. Riekel, Inst. Phys. Conf. Ser. 130, 523 (1992).

${ }^{3}$ J. Chavanne, P. Elleaume, E. Tarasona, Ya. M. Hartman, I. I. Snigireva, and A. A. Snigirev, Rev. Sci. Instrum. 65, 1959 (1994).

${ }^{4}$ A. Snigirev, I. Snigireva, C. Riekel, A. Miller, L. Wess, and T. Wess, J. Phys. IY 3, 443 (1993).

${ }^{5}$ S. M. Kuznetsov. I. I. Snigireva, A. A. Snigirev, P. Engström, and C. Riekel, Appl. Phys. Lett. 65, 1 (1994).

${ }^{6} \mathrm{~A}$. Snigirev, Rev. Sci. Instrum. (these proceedings).

${ }^{7}$ P. Chevallier, P. Dhez, F. Legrand, M. Idir, G. Soullie, A. Mirone, A. Erko, A. Snigirev, I. Snigireva, A. Suvorov, A. Freund, P. Engström, and A. Grübel, NIM (to be published). 